\title{
Enterprises’ Emission Reduction Cooperation in Carbon Supply Chain
}

\author{
Pan Xin-Yu ${ }^{1,2, *}$, Zhao Daozhi ${ }^{1}$ and Wu Cheng-Xia ${ }^{1}$ \\ ${ }^{1}$ College of Management and Economics, Tianjin University, Tianjin 300072, China; ${ }^{2}$ Department of Economics and \\ Management, Tangshan College, Tangshan 063000, China
}

\begin{abstract}
This paper investigates how upstream and downstream enterprises choose emission reduction strategy while they are facing environmental regulation, but also analyses their profits and reduction efficiencies in three typical situations. It can be obtained that in full-cooperation case, both their profit and reduction efficiency are optimal. Meanwhile, the relationship between reduction and carbon price can also be analyzed, which allows for externality and different carbon quota allocations. In brief, it can provide a theoretical basis for enterprise to make a wise strategy on emission reduction.
\end{abstract}

Keywords: Carbon price, carbon quota allocation policy, emission reduction rate, marginal utility, social welfare.

\section{INTRODUCTION}

From the Kyoto Protocol to the Doha Conference, the international community has paid more and more attention to the global climate change which has brought us the adverse consequences. The clean development mechanism, abbreviation as CDM, is one of the flexible compliance mechanisms of the" Kyoto Protocol".

China is one of CDM attachment countries, and its market has appeared a lot of carbon exchange centers, such as Shanghai carbon exchange center and Tianjin carbon exchange center. Also, there are many large enterprises which have begun the implementation of carbon emission reduction and trading. Consequently, carbon emission constraint will transform enterprise's cost structure and profit pattern, and then change enterprise's business behavior; but also in supply chain system, carbon emissions would create different marginal value for different enterprises.

\section{LITERATURE REVIEW}

Some scholars [1,2] have compared free distribution and auction patterns separately from the prospect of economic efficiency, environmental effectiveness, political acceptability, innovation driven and other aspects. Zhang [3] has showed that carbon emissions trading is very active in international scope. Liao \& Önal et al. [4] have researched on the relationship and nature of shadow price and equilibrium price in emissions trading market. Meng [5] has compared two technology policies that subsidizing emission reduction $R \& D$ and encouraging cooperation under the condition of exogenous emissions tax.

Du Shaofu [6] have studied the model of enterprise production optimization under the condition of deterministic demand considering carbon emissions trading mechanism. Zhang et al. [7] has established optimization decision model of production and storage under the mechanism of enterprise's dependence on carbon emissions trading by newsvendor model. While Hua et al. [8], focused on the problem of optimal order quantity which is only taken carbon emissions trading mechanism into account on the condition of deterministic demand.

Diabat \& Simchi-Levi [9] studied how to design supply chain factories and distribution centers to minimize cost and restrict carbon emissions. Cachon [10] has analyzed that how carbon footprint reduction to affect supply chain's operation. Benjaafar et al. [11] have analyzed how to reduce carbon emissions by means of operation decision. Cachon [12] has researched the problem of downstream retail branches layout optimization while facing carbon emission cost. Du et al. [13] has put forward a new kind of supply chain that regards traditional non-profit green environmental protection organizations as the suppliers of carbon emission permits.

We have noticed that Benjaafar, Cachon, Simchi-Levi, Fransoo, Ramudhin and Du Shaofu, Zhang Jingjiang have made a seminal contribution in supply chain design and operation under the constraint of carbon emissions. They have done some important researches. But the research about cooperation emission reduction strategy of interdependence upstream and downstream enterprises between supply chain is very seldom. This paper will supply the gap of these researches.

\section{SCENARIO ASSUMPTIONS AND PARAMETERS SETTING}

This essay considers two enterprises, i.e. upstream and downstream in a supply chain, conduct technology investment for emission reduction. Two enterprises must take emission reduction strategy as their choice variables. Enterprises may make choice between individual emission reduction and cooperative emission reduction. However, in differ- 
ent cooperation cases, two enterprises' emission reduction efficiencies, profits and total emission are not the same as well. We have to make such hypothesis as follow before we start to build up model.

1) The product is necessity and its market is not perfectly competitive market.

2) The product demand is linear function to price.

3) In a certain technical condition, carbon emission of unit product is constant, therefore, the enterprises' total carbon emissions will be a linear function to the output.

4) The emission reduction investment has no work upon the production cost of unit product.

5) Emission reduction cost is monotonically increasing function to emission reduction, and the marginal emission reduction cost will increase with the increasing of the emission reduction rate.

6) The carbon price is decided by carbon trading market; therefore, it is also an exogenous variable in this essay.

7) The government's single cycle distribution of carbon quotas is exogenous variable that cannot be transferred to the next stage.

(1) Notation:

$e_{i}$ : original carbon emissions of unit product, $\Delta e_{i}:$ reduced carbon emissions of unit product.

$\tau_{i}$ : emission reduction rate (that is $0 \leq \tau_{i}=\frac{\Delta e_{i}}{e_{i}}<1$ ), to some extent, it can be considered as the efficiency of carbon emission reduction.

$E_{i}(Q)$ : enterprise's total carbon emissions, $\left(E_{i}(Q)=e_{i} \cdot Q\right)$.

$C\left(\tau_{i}\right)$ : total cost of emission reduction. According to the above mentioned assumptions, there are $\left(C(0)=0, C(1)=+\infty, C^{\prime}\left(\tau_{i}\right)>0, C^{\prime \prime}\left(\tau_{i}\right)>0\right)$, in general, we can put this type of cost as the quadratic function to emission reduction rate, i.e. $C\left(\tau_{i}\right)=\frac{1}{2} m \cdot \tau_{i}^{2}$.

$E_{\text {trading }(i)}$ : trading amount of carbon emission permits in carbon market.

$Z_{i}$ : carbon quotas of government initial allocation, $Z_{i}$ is regarded as exogenous variable in this paper.

$C_{i}$ : production cost of unit product, $w$ : wholesale price; transfer price, $p_{c}$ : carbon price, $N$ : market capacity, $W$ : social welfare which does not consider external influence, $p$ : product price, $Q$ : product demand.

$U$ : abbreviation of upstream enterprise, $D$ : abbreviation of downstream enterprise,
$N C$ : Non-cooperation, $H C$ : Half-cooperation, $T C$ : Full-cooperation.

\section{The Model}

To an enterprise which conducts carbon emission reduction, its profit function is composed of three parts: sales revenue, investment of carbon emission reduction and trading amount of carbon emission permits. Therefore, the profit function of upstream enterprise is Eq. (1):

$\Pi_{U}=\overbrace{\left(w-c_{U}\right) \cdot Q}^{T R}-\overbrace{\frac{1}{2} m \cdot \tau_{U}^{2}}^{R C}-\overbrace{E_{\text {trading }(U)} \cdot p_{c}}^{T C}$

Considering carbon emission permit's allocation and trading, production-oriented enterprise's carbon quotas come from three channels: government initial allocation, the amount of carbon emission reduction and trading amount of carbon emission permits in the market. Meanwhile, the production-oriented enterprise's final carbon emissions will not exceed the total carbon quotas. In order to maximize enterprise's profit, enterprise should make full use of the resource of carbon quotas. That is Eq. (2):

$$
E_{\text {trading }(U)}=e_{U} \cdot Q-Z_{U}-\tau_{U} \cdot e_{U} \cdot Q
$$

By substitution Eq. (2) into Eq. (1), and the demand function, we can obtain Eq. (3):

$$
\Pi_{U}=\left[w-c_{U}-e_{U} \cdot p_{c} \cdot\left(1-\tau_{U}\right)\right] \cdot(N-b \cdot p)-\frac{1}{2} m \cdot \tau_{U}^{2}+Z_{U} \cdot p_{c}
$$

Similarly, the profit function of downstream enterprise is Eq. (4):

$$
\Pi_{D}=\left[p-w-c_{D}-e_{D} \cdot p_{c} \cdot\left(1-\tau_{D}\right)\right] \cdot(N-b \cdot p)-\frac{1}{2} m \cdot \tau_{D}^{2}+Z_{D} \cdot p_{c}
$$

Then, there will be two-stage game between upstream and downstream enterprises. The first stage is related with emission reduction investment, the second one is related with wholesale and retail price. Therefore, this two-stage game is divided into three forms. The first one is not completely cooperation, which is no cooperation on both product price and emission reduction investment completely; the second one is incomplete cooperation, namely they do not cooperate on product price but on emission reduction investment; the last one is full cooperation: the upstream and downstream enterprises have built a sufficient trust relationship.

(1) Non-Cooperation

Let

$\frac{\partial \Pi_{D}}{\partial p}=(N-b \cdot p)-b \cdot\left[p-w-c_{D}-e_{D} \cdot p_{c} \cdot\left(1-\tau_{D}\right)\right]$ equal to zero.

Substitute the results into Eq. (3) and let $\frac{\partial \Pi_{U}}{\partial w}$ equal to zero.

Eq. (5) can be obtained.

$Q_{N C}^{*}=\frac{N-b \cdot\left[c_{U}+c_{D}+e_{U} \cdot p_{c} \cdot\left(1-\tau_{U}\right)+e_{D} \cdot p_{c} \cdot\left(1-\tau_{D}\right)\right]}{4}$ 
Substitute Eq. (5) into the two enterprises' profit function, and then by solving the first order partial derivatives of the variable $\Pi_{i}$ with respect to independent variable $\tau_{i}$, let $\frac{\partial \Pi_{i}}{\partial \tau_{i}}(i=U, D)$ equal to zero.

Therefore, the enterprises' emission reduction rates can be obtained as follows.

$$
\left\{\begin{array}{l}
\tau_{U(N C)}^{*}=\frac{2 e_{U} \cdot p_{c} \cdot\left[N-b \cdot\left(c_{U}+c_{D}+e_{U} \cdot p_{c}+e_{D} \cdot p_{c}\right)\right]}{8 m-b \cdot p_{c}^{2} \cdot\left(e_{D}^{2}+2 e_{U}^{2}\right)} \\
\tau_{D(N C)}^{*}=\frac{e_{D} \cdot p_{c} \cdot\left[N-b \cdot\left(c_{U}+c_{D}+e_{U} \cdot p_{c}+e_{D} \cdot p_{c}\right)\right]}{8 m-b \cdot p_{c}^{2} \cdot\left(e_{D}^{2}+2 e_{U}^{2}\right)}
\end{array}\right.
$$

\section{(2) Half-Cooperation}

In the second stage, $\mathrm{HC}$ is the same decision-making strategy with non-cooperation pattern. Hence, the product price, demand and wholesale price are same as Eq. (5).

However, in the first stage, the upstream and downstream enterprises determine their own emission reduction rates. The whole supply chain's profits are as follows.

$$
\begin{aligned}
& \therefore \Pi_{(H C)}= \\
& \frac{3\left\{N-b \cdot\left[c_{U}+c_{D}+e_{U} \cdot p_{c} \cdot\left(1-\tau_{U}\right)+e_{D} \cdot p_{c} \cdot\left(1-\tau_{D}\right)\right]\right\}^{2}}{16 b}- \\
& \frac{1}{2} m \cdot\left(\tau_{U}^{2}+\tau_{D}^{2}\right)+\left(Z_{U}+Z_{D}\right) \cdot p_{c}
\end{aligned}
$$

By separately solving the first order partial derivatives to emission reduction rate, let them equal to zero. Then, the Hessen Matrix is as followed.

$$
H_{(H C)}=\left|\begin{array}{ll}
\frac{\partial^{2} \Pi_{(H C)}}{\partial \tau_{U}^{2}} & \frac{\partial^{2} \Pi_{(H C)}}{\partial \tau_{U} \cdot \partial \tau_{D}} \\
\frac{\partial^{2} \Pi_{(H C)}}{\partial \tau_{D} \cdot \partial \tau_{U}} & \frac{\partial^{2} \Pi_{(H C)}}{\partial \tau_{D}^{2}}
\end{array}\right|=\left|\begin{array}{ll}
\frac{3 b \cdot e_{U}^{2} \cdot p_{c}^{2}}{8}-m & \frac{3 b \cdot e_{U} \cdot e_{D} \cdot p_{c}^{2}}{8} \\
\frac{3 b \cdot e_{U} \cdot e_{D} \cdot p_{c}^{2}}{8} & \frac{3 b \cdot e_{D}^{2} \cdot p_{c}^{2}}{8}-m
\end{array}\right|
$$

Because the determinant of two order derivatives to emission reduction rate is more than zero, it has maximum value. So we can get the optimal reduction rates of the upstream and downstream enterprises in the pattern of halfcooperation.

$$
\left\{\begin{array}{l}
\tau_{U(H C)}^{*}=\frac{3 e_{U} \cdot p_{c} \cdot\left[N-b \cdot\left(c_{U}+c_{D}+e_{U} \cdot p_{c}+e_{D} \cdot p_{c}\right)\right]}{8 m-3 b \cdot p_{c}^{2} \cdot\left(e_{D}^{2}+e_{U}^{2}\right)} \\
\tau_{D(H C)}^{*}=\frac{3 e_{D} \cdot p_{c} \cdot\left[N-b \cdot\left(c_{U}+c_{D}+e_{U} \cdot p_{c}+e_{D} \cdot p_{c}\right)\right]}{8 m-3 b \cdot p_{c}^{2} \cdot\left(e_{D}^{2}+e_{U}^{2}\right)}
\end{array}\right.
$$

\section{(3) The third pattern-full-cooperation}

The profit of the whole supply chain is Eq. (9).

$$
\begin{aligned}
& \Pi_{(T C)}=\left\{p-\left[c_{U}+c_{D}+e_{U} \cdot p_{c} \cdot\left(1-\tau_{U}\right)+e_{D} \cdot p_{c} \cdot\left(1-\tau_{D}\right)\right]\right\} \cdot \\
& (N-b \cdot p)-\frac{1}{2} m \cdot\left(\tau_{U}^{2}+\tau_{D}^{2}\right)+\left(Z_{U}+Z_{D}\right) \cdot p_{c}
\end{aligned}
$$

Let $\frac{\partial \Pi_{(T C)}}{\partial p}$ equal to zero.

Therefore, Eq. (10) can be obtained as follows.

$$
p_{(T C)}^{*}=\frac{N+b \cdot\left[c_{U}+c_{D}+e_{U} \cdot p_{c} \cdot\left(1-\tau_{U}\right)+e_{D} \cdot p_{c} \cdot\left(1-\tau_{D}\right)\right]}{2 b}
$$

$Q_{(T C)}^{*}=\frac{N-b \cdot\left[c_{U}+c_{D}+e_{U} \cdot p_{c} \cdot\left(1-\tau_{U}\right)+e_{D} \cdot p_{c} \cdot\left(1-\tau_{D}\right)\right]}{2}$

By substituting Eq. (10) into Eq. (9), the optimal profit of the whole supply chain can be obtained. Because the determinant of two order derivatives to emission reduction rate is more than zero, it has maximum value.

$$
H_{(T C)}=\left|\begin{array}{cc}
\frac{\partial^{2} \Pi_{(T C)}}{\partial \tau_{U}^{2}} & \frac{\partial^{2} \Pi_{(T C)}}{\partial \tau_{U} \cdot \partial \tau_{D}} \\
\frac{\partial^{2} \Pi_{(T C)}}{\partial \tau_{D} \cdot \partial \tau_{U}} & \frac{\partial^{2} \Pi_{(T C)}}{\partial \tau_{D}^{2}}
\end{array}\right|=\left|\begin{array}{ll}
\frac{b \cdot e_{U}^{2} \cdot p_{c}^{2}}{2}-m & \frac{b \cdot e_{U} \cdot e_{D} \cdot p_{c}^{2}}{2} \\
\frac{b \cdot e_{U} \cdot e_{D} \cdot p_{c}^{2}}{2} & \frac{b \cdot e_{D}^{2} \cdot p_{c}^{2}}{2}-m
\end{array}\right|
$$

In the same way, we can get the optimal emission reduction rates of the upstream and downstream enterprises in the pattern of full-cooperation. That is Eq. (11).

$$
\left\{\begin{array}{l}
\tau_{U(T C)}^{*}=\frac{e_{U} \cdot p_{c} \cdot\left[N-b \cdot\left(c_{U}+c_{D}+e_{U} \cdot p_{c}+e_{D} \cdot p_{c}\right)\right]}{2 m-b \cdot p_{c}^{2} \cdot\left(e_{D}^{2}+e_{U}^{2}\right)} \\
\tau_{D(T C)}^{*}=\frac{e_{D} \cdot p_{c} \cdot\left[N-b \cdot\left(c_{U}+c_{D}+e_{U} \cdot p_{c}+e_{D} \cdot p_{c}\right)\right]}{2 m-b \cdot p_{c}^{2} \cdot\left(e_{D}^{2}+e_{U}^{2}\right)}
\end{array}\right.
$$

\section{Analysis and Discussion}

\section{(1) Carbon price's influence on emission reduction rate}

In order to simplify calculation process, now we hypothesis that $b$ equals to one, $c_{i}(i=U, D)$ equals to zero, and $e_{i}(i=U, D)$ equals to one. Then, Eq. (6), (8) and (11) can be converted into Eq. (12), (13) and (14) (In which, we assume that $\left.\bar{\tau}_{(i C)}^{*}=\bar{\tau}_{U(i C)}^{*}+\bar{\tau}_{D(i C)}^{*}\right)^{1}$. That is the supply chain total emission reduction rate is equal to the sum of the upstream and downstream enterprises' emission reduction rates.

\footnotetext{
${ }^{1}$ Supply chain reduction rate may not be the sum of individual enterprises emission reduction rate, it can be described by $\tau_{(i c)}^{*}=\frac{e_{U} \cdot \tau_{U(i c)}^{*}+e_{D} \cdot \tau_{D(i c)}^{*}}{e_{U}+e_{D}}$ accurately, but, because $e_{i}$ is a constant, reduction rate's linear calculation with $e_{i}$ cannot alter its linear performance. Therefore, $\bar{\tau}_{(i C)}^{*}=\bar{\tau}_{U(i C)}^{*}+\bar{\tau}_{D(i C)}^{*}$ will not change the performance of $\tau_{(i c)}^{*}=\frac{e_{U} \cdot \tau_{U(i c)}^{*}+e_{D} \cdot \tau_{D(i c)}^{*}}{e_{U}+e_{D}}$ in this context. Moreover, we have assumed that $e_{i}(i=U, D)$ equals to one. Here, we just research how carbon price influences on emission reduction rate in the three cases. However, from the amount of emission reduction point of view, the sum can be explained that if the arbitrary enterprise's reduction rate is high, the whole supply chain's amount of emission reduction is also high.
} 

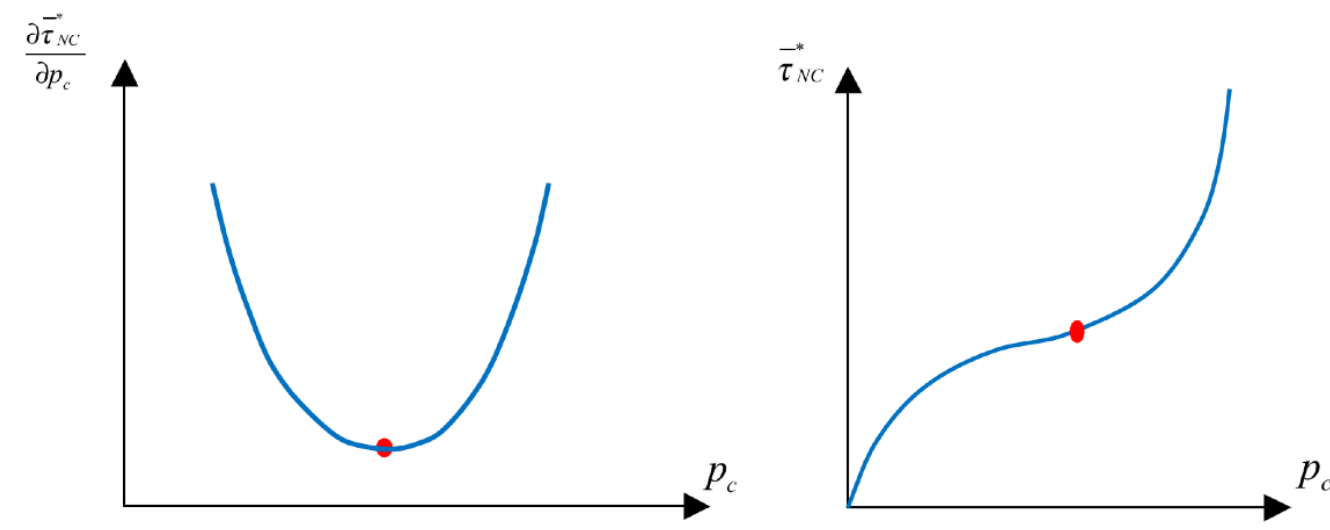

Fig. (1). When $32 m-3 N^{2}<0$, the relationship between $\frac{\partial \bar{\tau}_{N C}^{*}}{\partial p_{c}}, \tau_{N C}^{*}$ and $p_{c}$.

$$
\begin{aligned}
& \left\{\begin{array}{l}
\bar{\tau}_{U(N C)}^{*}=\frac{2 p_{c} \cdot\left(N-2 p_{c}\right)}{8 m-3 p_{c}^{2}} \\
\bar{\tau}_{D(N C)}^{*}=\frac{p_{c} \cdot\left(N-2 p_{c}\right)}{8 m-3 p_{c}^{2}} \\
\bar{\tau}_{(N C)}^{*}=\frac{3 p_{c} \cdot\left(N-2 p_{c}\right)}{8 m-3 p_{c}^{2}}
\end{array}\right. \\
& \left\{\begin{array}{l}
\bar{\tau}_{U(H C)}^{*}=\frac{3 p_{c} \cdot\left(N-2 p_{c}\right)}{8 m-6 p_{c}^{2}} \\
\bar{\tau}_{D(H C)}^{*}=\frac{3 p_{c} \cdot\left(N-2 p_{c}\right)}{8 m-6 p_{c}^{2}} \\
\bar{\tau}_{(H C)}^{*}=\frac{6 p_{c} \cdot\left(N-2 p_{c}\right)}{8 m-6 p_{c}^{2}}
\end{array}\right. \\
& \left\{\begin{array}{l}
\bar{\tau}_{U(T C)}^{*}=\frac{p_{c} \cdot\left(N-2 p_{c}\right)}{2 m-2 p_{c}^{2}} \\
\bar{\tau}_{D(T C)}^{*}=\frac{p_{c} \cdot\left(N-2 p_{c}\right)}{2 m-2 p_{c}^{2}} \\
\bar{\tau}_{(T C)}^{*}=\frac{p_{c} \cdot\left(N-2 p_{c}\right)}{m-p_{c}^{2}}
\end{array}\right.
\end{aligned}
$$

It can be illustrated that the optimal reduction rates, in the three patterns, have all been affected by the fluctuant of carbon price. Different carbon prices will lead to different emission reduction efficiencies.

$$
\begin{aligned}
& \text { 1) In non-cooperation case: } \\
& \frac{\partial \bar{\tau}_{(N C)}^{*}}{\partial p_{c}}=\frac{9 N \cdot p_{c}^{2}-96 m \cdot p_{c}+24 m \cdot N}{\left(8 m-3 p_{c}^{2}\right)^{2}}
\end{aligned}
$$

When $9 N \cdot p_{c}^{2}-96 m \cdot p_{c}+24 m \cdot N>0$, emission reduction rate and carbon price are positive correlation. Otherwise, they are negative correlation.
There will be no intersection point between the curve $\frac{\partial \bar{\tau}_{(N C)}^{*}}{\partial p_{c}}$ and the horizontal axis, because $\frac{\partial \bar{\tau}_{(N C)}^{*}}{\partial p_{c}}$ is always larger than zero. It explains that when emission reduction is relatively easy, emission reduction rate and carbon price are positive correlation. The reason is that if carbon price increases, enterprises will be more inclined to their own investment on emission reduction, the emission reduction rate will increase also. But if carbon price decreases, enterprises will buy carbon quotas from trading market, emission reduction rate will decrease also. Furthermore, when carbon price is relatively low, reduction rate will increase as carbon price increases, but the increment of it is getting smaller and smaller, mainly due to the diminishing marginal utility of reduction investment. Their relationship is shown in Fig. (1).

If $\Delta=32 m-3 N^{2}>0$, it illustrates that $m$ is larger and emission reduction is not easy to realize. There are two intersection points between the curve $\frac{\partial \bar{\tau}_{(N C)}^{*}}{\partial p_{c}}$ and the horizontal axis. There are $p_{c}^{-}=\frac{16 m-2 \sqrt{64 m^{2}-6 N^{2} \cdot m}}{3 N}$ and $p_{c}^{+}=\frac{16 m+2 \sqrt{64 m^{2}-6 N^{2} \cdot m}}{3 N}$. It can be seen from the parabola that, when carbon price is located in the section of $\left(0, p_{c}^{-}\right)$, emission reduction rate will increase as carbon price increases. Nevertheless, the increment of reduction rate will decrease with carbon price increasing. That is because when carbon price is relatively low, its tiny increment will make enterprise think to obtain carbon quotas through their own emission reduction. But the increment of reduction rate is gradually decreasing due to the diminishing marginal utility of emission reduction. When carbon price equals to $p_{c}^{-}$, the variance ratio of reduction rate is equal to zero. At this time, more investment on emission reduction will not produce any changes to the emission reduction rate; it will reach a maxi- 

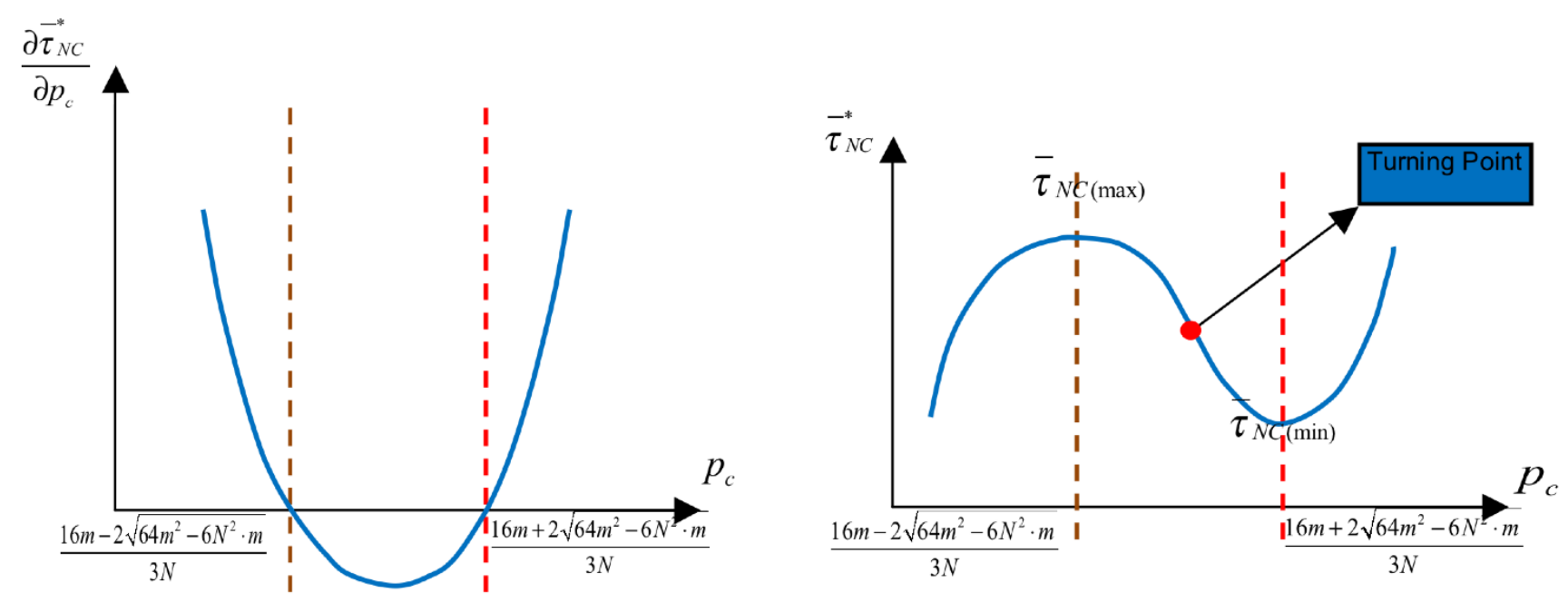

Fig. (2). When $32 m-3 N^{2}>0$, the relationship between $\frac{\partial \tau_{N C}^{*}}{\partial p_{c}}, \tau_{N C}^{*}$ and $p_{c}$.

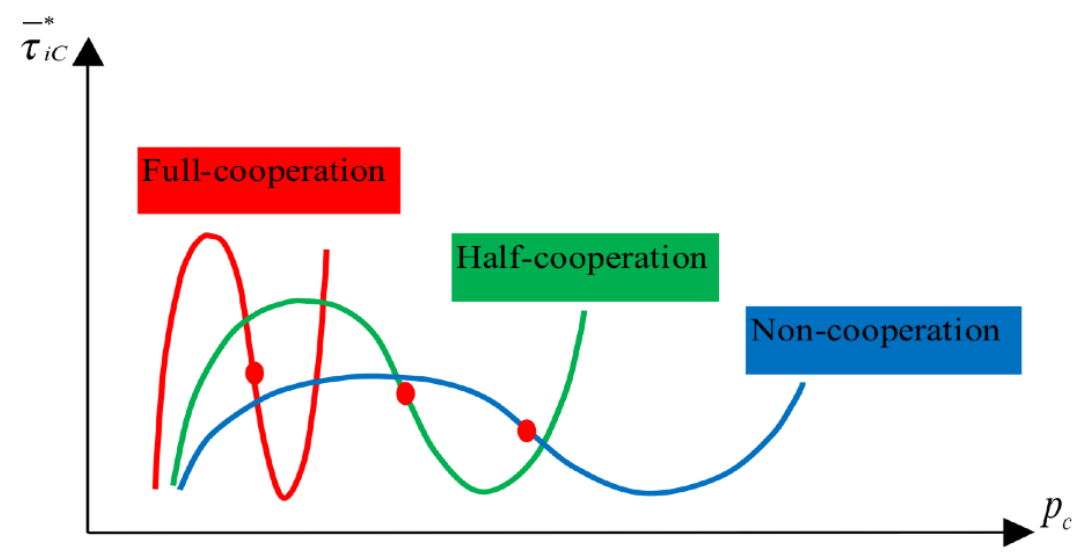

Fig. (3). Relationship between reduction rate and carbon price in the three cases (when $\Delta>0$ ).

mum value. When carbon price belongs to the section of $\left(p_{c}^{-}, p_{c}^{+}\right)$, because reduction investment has no effect and the difficulty of reduction is very high, enterprises will abandon their own emission reduction but consider buying required carbon quota from carbon trading market. That is Fig. (2).

\section{2) In half-cooperation case:}

If $\Delta=16 m-3 N^{2}<0$, the relationship between carbon price and emission reduction is positive correlation. And if $\Delta=16 m-3 N^{2}>0$, they would appear a negative correlation region. The reason is no different to the non-cooperation case. Only the slope and the range of the curve are disparate.

\section{3) In full-cooperation case:}

Similarly, if $\Delta=4 m-N^{2}<0$, the relationship between carbon price and emission reduction is positive correlation. Otherwise, if $\Delta=4 m-N^{2}>0$, they would appear a negative correlation region. The reason is no different to the noncooperation case. Only the slope and the range of the curve are disparate. Up to the present, we can summarize the above results in Fig. (3).
From Fig. (3), we can see that negative correlation area gradually becomes smaller along with the degree of cooperation enhancing. And full-cooperation case's suitable carbon price area where the optimal reduction rate can be accomplished may be smaller than non-cooperative case's. Furthermore, it can be deduced that full-cooperation enterprises will be more adapted to a lower carbon price. That is likely to occur that under the same carbon price, cooperative enterprises' emission reduction rate is reducing while non cooperative enterprises' emission reduction rate is increasing; or cooperative enterprises' emission reduction rate is increasing while non cooperative enterprises' emission reduction rate is reducing.

Also, we can get that in full-cooperation condition, small change of carbon price will cause a great change in supply chain's reduction rate, but in non-cooperation condition, its bigger change will only cause a small change in supply chain's reduction rate. Along with the carbon trading market gradually becoming mature, carbon price will tend to be stable, then some non cooperative enterprises that cannot adapt to this price will not achieve a good reduction effect, meanwhile, other cooperative enterprises that can adapt to this price will accomplish a greater reduction effect. 


\section{(2) Comparison of emission reduction rate}

Through Eq. (6), (8) and (11), the difference value of emission reduction rate can be obtained.

$$
\begin{aligned}
& \because \tau_{U(H C)}^{*}-\tau_{U(N C)}^{*}= \\
& \frac{\left(8 m+3 b \cdot e_{D}^{2} \cdot p_{c}^{2}\right) e_{U} \cdot p_{c} \cdot\left[N-b \cdot\left(c_{U}+c_{D}+e_{U} \cdot p_{c}+e_{D} \cdot p_{c}\right)\right]}{\left[8 m-b \cdot p_{c}^{2} \cdot\left(e_{D}^{2}+2 e_{U}^{2}\right)\right] \cdot\left[8 m-3 b \cdot p_{c}^{2} \cdot\left(e_{D}^{2}+e_{U}^{2}\right)\right]}>0 \\
& \because \tau_{U(T C)}^{*}-\tau_{U(H C)}^{*}= \\
& \text { And } \frac{2 m \cdot e_{U} \cdot p_{c} \cdot\left[N-b \cdot\left(c_{U}+c_{D}+e_{U} \cdot p_{c}+e_{D} \cdot p_{c}\right)\right]}{\left[2 m-b \cdot p_{c}^{2} \cdot\left(e_{D}^{2}+e_{U}^{2}\right)\right] \cdot\left[8 m-3 b \cdot p_{c}^{2} \cdot\left(e_{D}^{2}+e_{U}^{2}\right)\right]}>0 \\
& \therefore \tau_{U(T C)}^{*}>\tau_{U(H C)}^{*}>\tau_{U(N C)}^{*} \\
& \therefore \tau_{D(T C)}^{*}>\tau_{D(H C)}^{*}>\tau_{D(N C)}^{*}
\end{aligned}
$$

Therefore, full-cooperation situation' emission reduction rates are the highest, followed by half-cooperation, and noncooperation. We can also deduce that cooperative supply chain's emission reduction effect is better than noncooperative supply chain's.

\section{(3) Total carbon emissions in three different cases}

By means of the above analysis, we can get the supply chain's total outputs in the three cases. It can be obtained from the comparison that:

$$
\begin{aligned}
& \because Q_{(T C)}^{*}-Q_{(H C)}^{*}= \\
& \frac{m \cdot\left[N-b \cdot\left(c_{U}+c_{D}+e_{U} \cdot p_{c}+e_{D} \cdot p_{c}\right)\right]}{\left[2 m-b \cdot p_{c}^{2} \cdot\left(e_{D}^{2}+e_{U}^{2}\right)\right] \cdot\left[8 m-3 b \cdot p_{c}^{2} \cdot\left(e_{D}^{2}+e_{U}^{2}\right)\right]} \cdot[4 m- \\
&\left.b \cdot p_{c}^{2}\left(e_{D}^{2}+e_{U}^{2}\right)\right]>0 \\
& \because Q_{(T C)}^{*}-Q_{(H C)}^{*}= \\
& \frac{2 m \cdot\left[N-b \cdot\left(c_{U}+c_{D}+e_{U} \cdot p_{c}+e_{D} \cdot p_{c}\right)\right]}{\left[8 m-b \cdot p_{c}^{2} \cdot\left(e_{D}^{2}+2 e_{U}^{2}\right)\right] \cdot\left[8 m-3 b \cdot p_{c}^{2} \cdot\left(e_{D}^{2}+e_{U}^{2}\right)\right]} \cdot b \cdot p_{c}^{2} \\
&\left(2 e_{D}^{2}+e_{U}^{2}\right)>0 \\
& \therefore Q_{(T C)}^{*}>Q_{(H C)}^{*}>Q_{(N C)}^{*}
\end{aligned}
$$

Under the premise of pursuing the goal of profit maximization, the total supply chain's output increases gradually along with the degree of cooperation rising ceaselessly. We will calculate supply chain's carbon emissions of unit product in the three cases. Through comparison, we can easily get

$$
e_{(T C)}^{*}<e_{(H C)}^{*}<e_{(N C)}^{*}
$$

From above analysis, we can see that in full cooperation case, unit product's emission is the smallest, but the total emissions may be out of comparison because of the biggest output. It is determined by different market situations and other parameters. That is to say, although unit product's emission reduction effect is best, the expanding demand leads to increase the total emissions invisibly. Hence, in order to control total emissions to increase under the premise of ensuring emission reduction rate unchanged, the best way is to decrease enterprise's output. Therefore, we can say that it is an irreconcilable contradiction between the whole supply chain's profits and overall carbon emissions. In order to make total carbon emissions less than government's emission limitation, enterprises have to sacrifice a portion of their profits; but if they pursuit the maximization of their profits, there will be more carbon emissions.

\section{(4) Supply chain's profit in the three cases}

We put above results into the upstream and downstream enterprises' profit function in the three conditions.

$$
\begin{aligned}
& \Pi_{(H C)}^{*}-\Pi_{(N C)}^{*}=m \cdot b \cdot p_{c}^{2} \cdot\left(e_{U}^{2}+2 e_{D}^{2}\right) \cdot\left[40 m-3 b \cdot p_{c}^{2} \cdot\left(e_{U}^{2}+e_{D}^{2}\right)\right] \cdot \\
& {\left[4 m-3 b \cdot p_{c}^{2} \cdot\left(e_{U}^{2}+e_{D}^{2}\right)\right]} \\
& \cdot \frac{\left[N-b \cdot\left(c_{U}+c_{D}+e_{U} \cdot p_{c}+e_{D} \cdot p_{c}\right)\right]^{2}}{\left[8 m-3 b \cdot p_{c}^{2} \cdot\left(e_{U}^{2}+e_{D}^{2}\right)\right]^{2} \cdot\left[8 m-b \cdot p_{c}^{2} \cdot\left(2 e_{U}^{2}+e_{D}^{2}\right)\right]^{2}} \\
& \quad \because 4 m-3 b \cdot p_{c}^{2} \cdot\left(e_{U}^{2}+e_{D}^{2}\right)>0^{2} \\
& \quad \therefore \Pi_{(H C)}^{*}>\Pi_{(N C)}^{*} \\
& \Pi_{(T C)}^{*}-\Pi_{(H C)}^{*}=4 m^{2} \cdot\left[m-b \cdot p_{c}^{2} \cdot\left(e_{U}^{2}+e_{D}^{2}\right)\right] \cdot \\
& \quad\left[4 m-3 b \cdot p_{c}^{2} \cdot\left(e_{U}^{2}+e_{D}^{2}\right)\right] \cdot \\
& \quad \frac{\left[N-b \cdot\left(c_{U}+c_{D}+e_{U} \cdot p_{c}+e_{D} \cdot p_{c}\right)\right]^{2}}{\left[8 m-3 b \cdot p_{c}^{2} \cdot\left(e_{U}^{2}+e_{D}^{2}\right)\right]^{2} \cdot\left[2 m-b \cdot p_{c}^{2} \cdot\left(e_{U}^{2}+e_{D}^{2}\right)\right]^{2}} \\
& \quad \because m-b \cdot p_{c}^{2} \cdot\left(e_{U}^{2}+e_{D}^{2}\right)>0 \text { and } 4 m-3 b \cdot p_{c}^{2} \cdot\left(e_{U}^{2}+e_{D}^{2}\right) \\
& >0 \quad \therefore \quad \Pi_{(T C)}^{*}>\Pi_{(H C)}^{*}
\end{aligned}
$$

Therefore, we can reach the conclusion that

$$
\Pi_{(N C)}^{*}<\Pi_{(H C)}^{*}<\Pi_{(T C)}^{*}
$$

As for a low carbon supply chain, full-cooperation between enterprises can reach the biggest profit, followed by their half-cooperation, and then smallest one is noncooperation.

\section{CONCLUSION}

Full-cooperation can achieve the most emission reduction efficiency for a single company in a supply chain; meanwhile it can get the maximum profit also. We should not only firstly take aim at the enterprises which unit product's carbon emissions are more than others, but also reduce carbon emission from the source of the whole supply chain. In the light of sensitivity analysis to carbon price, enterprises would fall into a zone where the relationship between reduction and carbon price is negative correlation, and then each enterprise should strengthen its cooperation and resist the difficulty of emission reduction and unstable carbon price. Only in this way can we obtain a better effect of emission reduction in the future low-carbon economic tide.

2 In half-cooperation case, downstream enterprise's profit is greater than zero, it must be in accord with $\frac{4 m^{2}}{b}-\frac{9}{2} m \cdot e_{D}^{2} \cdot p_{c}^{2}>0$. 


\section{CONFLICT OF INTEREST}

The authors confirm that this article content has no conflict of interest.

\section{ACKNOWLEDGEMENTS}

Declared none.

\section{REFERENCES}

[1] Sijm, J.P.M., M.M. Berk, M.G.J. den Ezlen, et.al. Options for Post-2012 EU Burden Sharing and EUETS Allocation. Bilthoven: Netherlands Environmental Assessment Agency, 2007.

[2] Cramton, P., S. Kerr. Tradeable Carbon Permit Auctions: How And Why to Auction Not Grandfather. Energy Policy, 2002, 30(4): 333-345.

[3] Zhang Zhongxiang. The Economic Impact of Emissions Trading Market-Based on 12 Countries and Regions Marginal Abatement Cost Global Model Analysis. The Journal of Quantitative \& Technical Economics, 2003, (9):95-99.

[4] Liao, C., H. Önal, M.H. Chen. Average Shadow Price and Equilibrium Price: Aase Study of Tradable Pollution Permit Markets. European Journal of Operational Research, 2009, 196(3): 12071213.
[5] Meng Weijun, Comparation of Subsidy and Cooperation Policy Based on Emission Reduction R\&D, systems engineering, Vol.28, No.11 Nov.,2010, p123-126.

[6] Du Shaofu, Dong Junfeng. Production Optimization Considering Emission Permits and Trading. Chinese Journal of management science, 2009,17(3):81-86.

[7] Zhang, J., T. Nie, S. Du. Optimal Emission-dependent Production Policy with Stochastic demand. Journal International Journal of Society Systems Science. 2011, 3(1-2): 21-39.

[8] Hua, G., T.C.E. Cheng, S. Wang. Managing Carbon Footprints in Inventory Management. International Journal of Production Economics. 2011, 132(2): 178-185.

[9] A Carbon-Capped Supply Chain Network Problem. IEEE International Conference on Industrial Engineering and Engineering Management, 2009. IEEM : 523-527.

[10] Cachon, G.P. Carbon Footprint and the Management of Supply Chains. The Informs Annual Meeting, San Diego: 2009.

[11] Benjaafar, S., Y. Li, M. Daskin. Carbon Footprint and the Management of Supply Chains: Insights from Simple Models. Working $\quad 2009$ http://www.ie.umn.edu/faculty/faculty/pdf/beyada-3-31-10.pdf.

[12] Cachon, G.P. Supply Chain Design and the Cost of Greenhouse Gas Emissions. Working paper: University of Pennsylvania, 2011

[13] Du, S., F. Ma, Z. Fu, et al. Game-theoretic Analysis for an Emission-dependent Supply Chain in a "cap-and-trade" System. Annals of Operations Research. 2011. DOI 10.1007/s10479-011 0964-6.

(C) Xin-Yu et al.; Licensee Bentham Open.

This is an open access article licensed under the terms of the Creative Commons Attribution Non-Commercial License (http://creativecommons.org/licenses/by-nc/3.0/) which permits unrestricted, non-commercial use, distribution and reproduction in any medium, provided the work is properly cited. 